\title{
Intra-Abdominal Hemorrhage, CTCAE
}

National Cancer Institute

\section{Source}

National Cancer Institute. Intra-Abdominal Hemorrhage, CTCAE. NCI Thesaurus. Code C143595.

A disorder characterized by bleeding in the abdominal cavity. 